Proceedings

\title{
Force-Sensing Hangboard to Enhance Finger Training in Rock Climbers ${ }^{+}$
}

\author{
Michael Anderson \\ Department of Engineering Mechanics, United States Air Force Academy, USAF Academy, CO 80840, USA; \\ michael.anderson@usafa.edu (M.A.); Tel.: +01-719-333-4048 \\ + Presented at the 12th conference of the International Sports Engineering Association, Brisbane, Queensland, \\ Australia, 26-29 March 2018.
}

Published: 13 February 2018

\begin{abstract}
In rock climbing, finger strength is directly related to performance. Here, a novel device is described to enhance finger strength training or aid in rehabilitation of finger injuries. The device incorporates load cells into an existing hangboard to measure finger force, record it and display it to the athlete in real time. The device was characterized for accuracy, linearity, hysteresis and repeatability, and found to have a resolution of $\pm 01.5 \mathrm{~N}$, sample rate of $10 \mathrm{~Hz}$, and linearity of 0.9998 . Preliminary athlete trials of the device verified its ability to more accurately record training exertion, that biases exist between the right and left hands of climbers and that this real-time performance feedback can improve training quality.
\end{abstract}

Keywords: rock climbing; training; finger strength; hangboard; rock prodigy training center

\section{Introduction}

Finger strength is critical in rock climbing, and is directly related to performance [1,2]. Climbers improve grip strength by hanging statically (often with additional weight attached) from a hangboard, a device with artificial grips. Finger strength training at an elite level, after years of training, requires a very high level of effort by the athlete to continue to make gains. The finger flexors undergo 7-s long isometric contractions during multiple repetitions, or "hangs". This is in contrast to typical strength training which involve eccentric-concentric muscle movements lasting only a few seconds or less. Therefore, effective training demands strong focus and concentration by the athlete, often training in isolation without motivational stimuli such as competition. As a result, the quality of this finger strength training is highly dependent on the athlete's mental stamina and control. Therefore, hangboard finger training might be enhanced by providing the athlete real-time feedback of finger force exertion during training.

Previous researchers have created sport-specific devices to measure finger strength [3,4], but these have been limited to measuring a single grip position, did not provide real-time feedback and were too complex for every-day training. These devices were developed to support exercise physiology research by collecting performance data on research subjects. Developed and presented here is a portable and robust instrumented training system that athletes can use in their homes. The intent of the device is not exclusively as a scientific instrument to evaluate training protocols, but also as a training tool to improve the quality of finger strength training. It is anticipated that providing real-time feedback to the athlete during training will improve the athlete's concentration, and thus, effort-resulting in better quality training. 


\section{Materials and Methods}

\subsection{The Force Board}

The Force Board system in Figure 1 has three main components; (1) a two-piece hangboard, such as the Trango "Rock Prodigy Training Center" or "Rock Prodigy Forge" [5-7]; (2) two load-sensing beams that interface with each piece of the hangboard; and (3) a microcontroller to condition, log and display the sensor data. The two-piece hangboard allows for individual sensing and recording of grip force from each hand. The hangboard has an assortment of artificial grips designed to simulate the most-challenging grips encountered on elite rock climbs. These include various sized edge, pocket, sloper and pinch grips. By using hangboards as a part of the Force Board system, it is possible to measure athlete performance on this complete range of climbing grips. Each hangboard piece is fitted with steel hooks that fit over the load-sensing beams, transferring the finger force of the athlete onto the load-sensing beams.

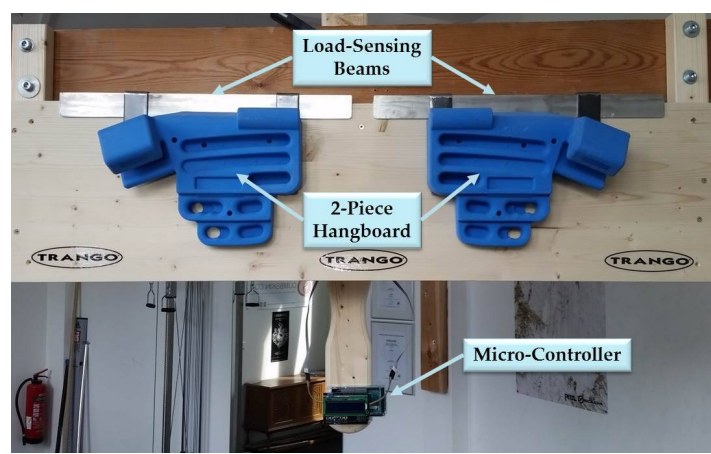

(a)

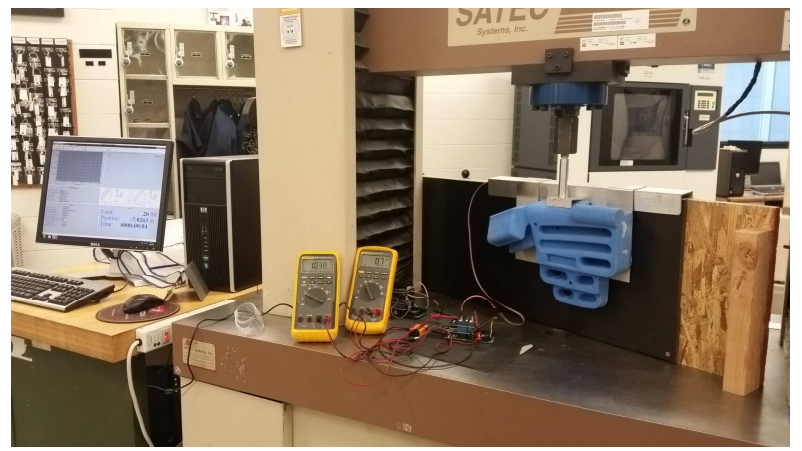

(b)

Figure 1. (a) The Force Board system indicating the component parts. This installations is in Kafe Craft Climbing Center, Nuremburg, Germany; (b) Characterizing sensor system performance in a laboratory load frame. The raw strain gauge voltage and amplified voltage are measured and recorded, and compared to the load applied by the SATEC machine.

Each load-sensing beam system (Figure 2a) consists of a 50-cm long C-channel that fits over two Sparkfun SEN-10245 load cells-one at each end of the channel. These are held in place by a thin beam. The load cells are steel cantilever beams that deflect in response to vertically applied forces. The two cantilever load cells (one underneath each end of the C-channel) are instrumented with strain gauges which are connected in a full Wheatstone bridge (Figure $2 b$ ) such that the strain from the two cells are summed. Therefore, regardless of the horizontal position of the hangboard along the Cchannel, the Wheatstone bridge will return strain that is proportional to the total finger force applied by the athlete from that hand.

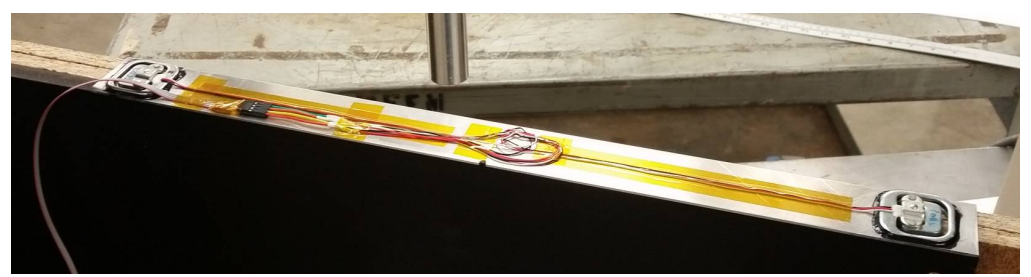

(a)

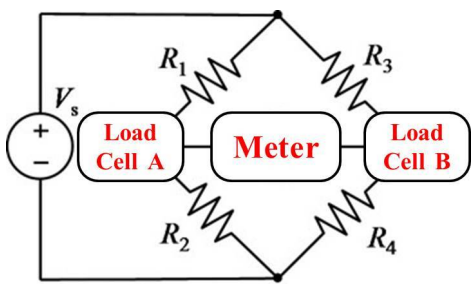

(b)

Figure 2. (a) The Load Sensing Beam, (b) The Wheatstone bridge circuit configuration.

The strain from each hangboard/load-beam set is measured by an Arduino Mega microcontroller and associated signal conditioning and software. A Robot Shop Arduino Strain Amplifier Shield is used to provide the precisely controlled $3.3 \mathrm{~V}$ excitation voltage to the two Wheatstone Bridges and sense the bridge voltages. The amplifier shield was modified to achieve a gain of 200, and then filtered by a 2nd order low-pass Bessel filter at $1000 \mathrm{~Hz}$. The force data is recorded onto an 
SD card with a Seeed Studio V4.0 Arduino SD shield and is displayed to the athlete in real-time via an Adafruit $16 \times 2$ LCD screen. This system is capable of measuring applied finger force from each hand with a resolution of $\pm 1.5 \mathrm{~N}( \pm 0.15 \mathrm{~kg})$ at a frequency of $10 \mathrm{~Hz}$. The resolution is limited by the 10 bit digital resolution (i.e., 1024 levels) of the analog-to-digital converter of the Arduino Mega microcontroller.

\subsection{Characterizing the Force Board}

To verify sensor performance and establish its functional range, the Force Board System was tested in a SATEC Load frame as shown in Figure 1b. Various regimes of known loads were applied and compared to the measurements recorded by the Force Board System.

The results of these tests are shown in Figure 3. The load cells have non-zero gauge voltages at zero load as a result of the Wheatstone Bridge balancing process, but these are accounted for during the tare process. All load cells exhibit excellent linearity over the expected measurement range of approximately $175-600 \mathrm{~N}$. This range is determined from typical rock climber mass plus or minus weight added or removed during training (referred to as "Weight-Hanging Ability" -WHA). Previous research indicated that this range of WHA is typical for a wide range of climbers [2]. The performance of Load Cell 1 was linear curve-fitted, and the $\mathrm{R}^{2}$ correlation between the measured data and a linear approximation is 0.9998 , indicating excellent linearity.

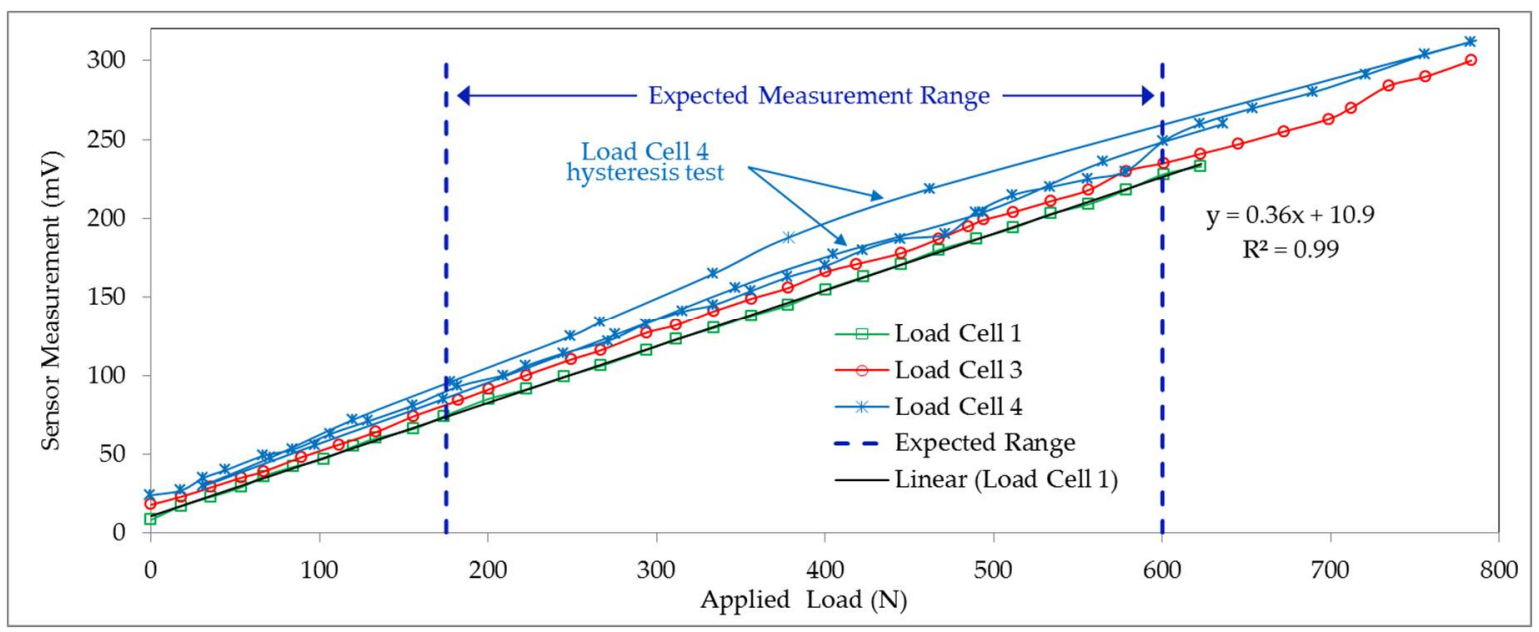

Figure 3. Force Board characterization results indicating linearity, repeatability and hysteresis.

Load Cell 4 was tested for hysteresis and repeatability by ramping up the applied load to $780 \mathrm{~N}$, returning to zero, and then ramping back up to $640 \mathrm{~N}$. Hysteresis is evident during unloading, but the sensor measurements were repeatable during loading. Due to the structural limitations of the load cells, they have a maximum working load of $50 \mathrm{~kg}(\sim 500 \mathrm{~N})$ each.

\subsection{Evaluation Methods}

After characterizing the Force Board sensing system, it was necessary to evaluate its influence on athletes during finger strength training. There were 3 goals of this initial evaluation; (1) Observe the accuracy of the athletes' "pen \& ink" measurement and record keeping of their exercises; (2) Observe differences in exertion between the right and left hands; (3) Determine if real-time exertion feedback provided by the digital display influenced the athletes' training. To evaluate these behaviors, the device was used by climbers during an existing training session. Two experienced and high-level athletes participated in the evaluation, one male and one female. The subjects were accustomed to training on the Trango Rock Prodigy Training Center and Forge hangboards and they were not directed to explicitly alter their training protocol in any way; they would perform the same grip positions with the same number, length and duty cycle of repetitions and sets. 
The subjects used the device for two different grip positions which included 3 sets per grip (for a total of 6 sets, and 36 repetitions). During the first set of each grip (the first 7 repetitions), the display screen was masked so that the subjects could not see their force feedback, then it was unmasked. The reason for this procedure was to determine if the athlete's behavior was altered by the availability of real-time force feedback.

\section{Results}

A typical force profile of an athlete completing the test protocol (2 grips, 6 sets, 36 reps) is shown in Figure 4. Table 1 details the accuracy with which athletes record their performance (loads and repetition duty cycle). Overall, the athlete's records are accurate; however, in the last sets of each grip, when exertion is maximal, the athlete's record diverges from the measurement. These errors are most apparent in the record of rest taken between repetitions.

Next, the variance between left vs. right hand effort is considered. Figure 4 and Figure 5 clearly show that the right hand is delivering approximately $10 \%$ greater force. Both athletes are righthanded, so this result might be expected.

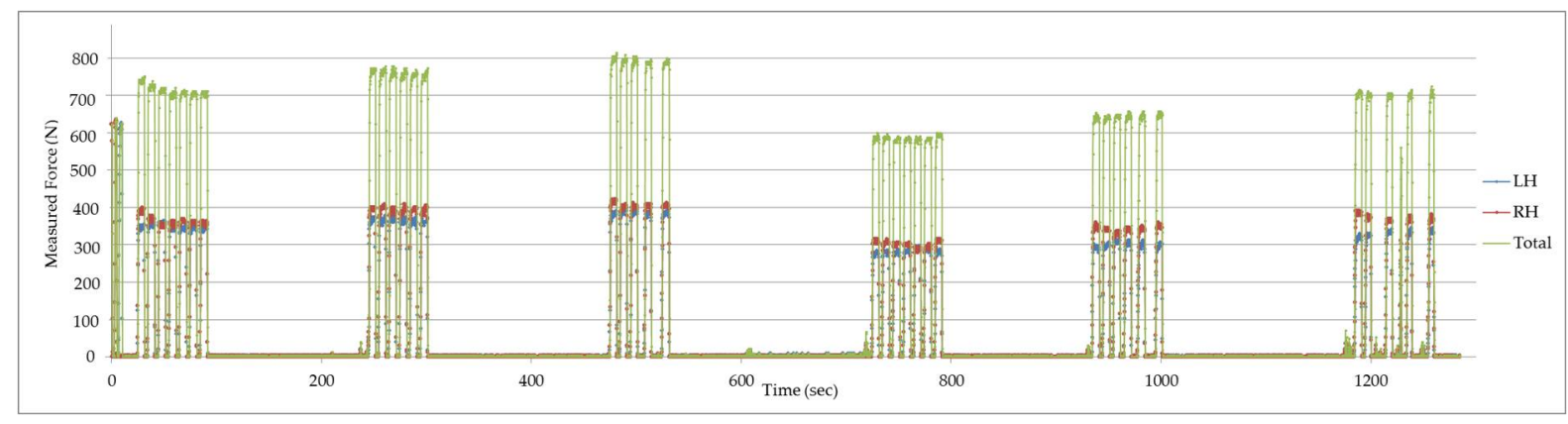

Figure 4. Force Measurements for "athlete 1". This displays 2 grips, 3 sets each (6 total sets) and 18 total repetitions per grip (36 reps total).

Table 1. Force Board measurement comparison to the athlete's records. Color indicates the athlete error is more than $10 \%$. If green, the athlete under-reported his/her effort, if red, he/she exaggerated the effort.

\begin{tabular}{lrrrrrrrrrrrr}
\hline & \multicolumn{4}{c}{ Repetition Average Duration (sec) } & \multicolumn{4}{c}{ Rest Average Duration (sec) } \\
\hline & Set 1 & Set 2 & Set 3 & Set 4 & Set 5 & Set 6 & Set 1 & Set 2 & Set 3 & Set 4 & Set 5 & Set 6 \\
\hline Measurement 1 & 6.96 & 6.95 & 6.92 & 7.19 & 7.01 & 5.43 & 2.98 & 3.06 & 5.55 & 2.79 & 5.00 & 7.04 \\
\hline Athlete 1 Record & 7 & 7 & 7 & 7 & 7 & 6 & 3 & 3 & 3 & 3 & 4 & 7.25 \\
\hline \% Error 1 & $1 \%$ & $1 \%$ & $1 \%$ & $-3 \%$ & $0 \%$ & $10 \%$ & $1 \%$ & $-2 \%$ & $-85 \%$ & $7 \%$ & $-25 \%$ & $3 \%$ \\
\hline Measurement 2 & 7.24 & 7.90 & 7.52 & - & - & - & 2.84 & 3.83 & 4.39 & - & - & - \\
\hline Athlete 2 Record & 7 & 7 & 7 & - & - & - & 3 & 3 & 3 & - & - & - \\
\hline \% Error 2 & $-3 \%$ & $-13 \%$ & $-7 \%$ & - & - & - & $5 \%$ & $-28 \%$ & $-46 \%$ & - & - & - \\
\hline
\end{tabular}




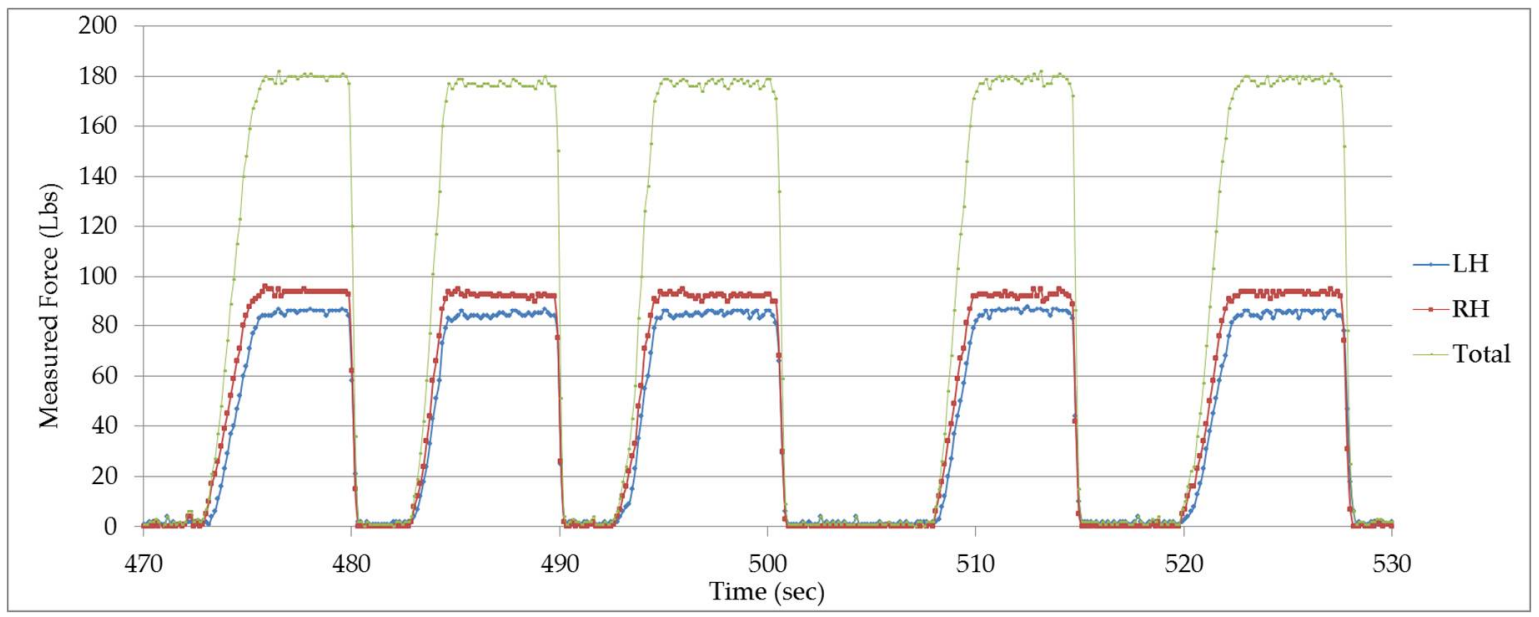

Figure 5. Athlete 2, set 3 indicating the exertion variance between left (LH) and right (RH) hands.

Finally, the first and second sets of each grip for athlete 2 are shown in Figure 6 which evaluated the effect of real-time feedback provided to the athlete. The display screen was masked during each of the first sets, then unmasked for the second set. If the total force shown by the green lines is considered, it is apparent that the athlete's exertion is more consistent when real-time feedback is provided.

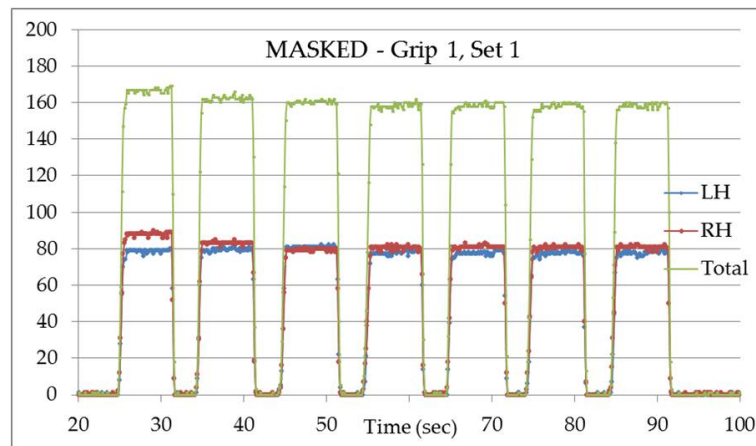

(a)

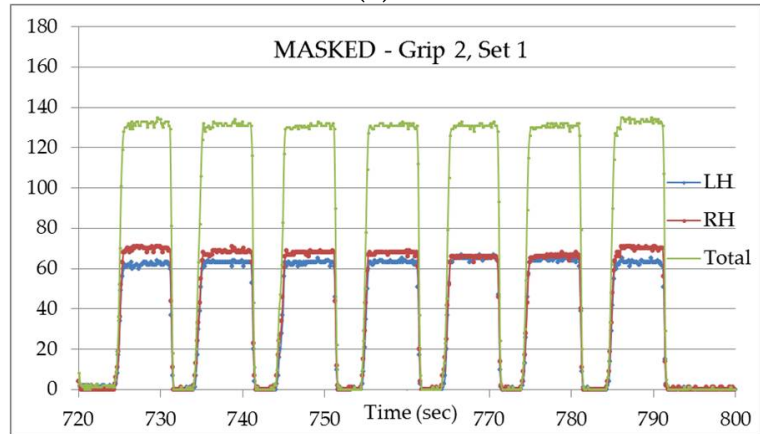

(c)

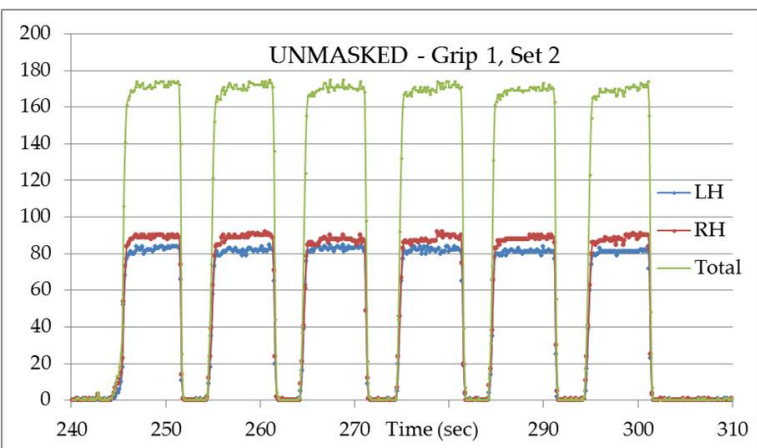

(b)

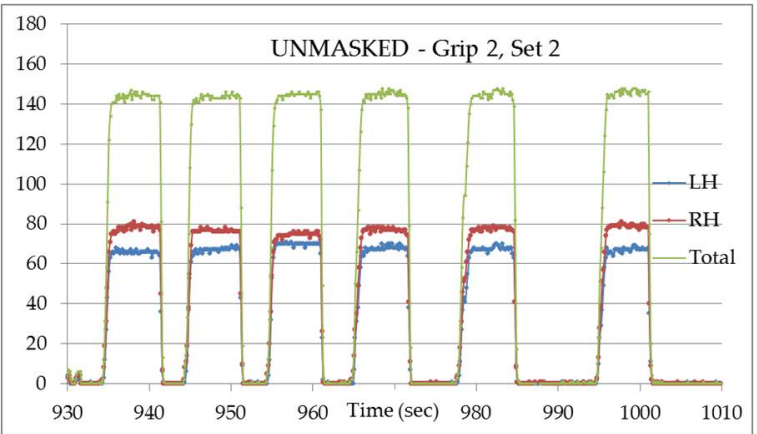

(d)

Figure 6. Exertion measurements for athlete 2. The ordinate axis is force, as in Figure 5. On the left, feedback to the athlete is masked-(a) Grip 1, Set 1 and (c) Grip 2, Set 1. On the right, feedback to the athlete is unmasked-(b) Grip 1, Set 2 and (d) Grip 2, Set 2.

\section{Discussion}

The purpose of this work is to report on the development and preliminary evaluation of a force sensing exercise device for climbers. It is expected that real-time feedback, especially if incorporated with audible cues, will motivate athletes to give increased effort and to more precisely execute the workout. Furthermore, the data obtained will enable coaches and/or physical therapists to modify training as needed to correct imbalances or other weaknesses. The results in Table 1 show that the 
Force Board can more accurately record the athletes' workout. This also presents an opportunity to couple these measurements with real-time virtual coaching, such as encouraging the athlete to continue exertion to the completion of all of the reps, rather than ending prematurely.

The variation between right and left hand exertion was also noted, and identified in Figure 5. In climbing, it has long been suspected that finger strength imbalances existed, yet sport-specific evidence was lacking. This experiment demonstrates this imbalance clearly, and identifies an opportunity for additional training or modified protocols to improve climber performance.

Finally, it is evident that providing real-time feedback to an athlete during training affects his/her exertion. This result is expected, and will be exploited and evaluated much more thoroughly in future experiments. Indeed, the purpose in developing the device was to influence athlete exertion, so this feature needs to be exploited. Future experiments will endeavor to use the device with numerous athletes over entire training cycles (encompassing 10 or more workouts) to determine the long-term results of training with real-time feedback.

In addition, Force Board will be used in future work to measure the rate of force development (RFD), which is currently of very high interest in the climbing research community. High RFD is critical in climbing, identified as one of the top three determinants of performance [8]. Currently, the Force Board is the only device in the literature able to measure RFD during sport-specific training. This awareness should allow researchers to develop enhanced training protocols to improve RFD in climbers, and thus, vastly improve athlete performance.

\section{Conclusions}

This work presented the design, development and testing of a novel device, the Force Board, to measure finger force developed by climbers during training, and provide the data to the athlete in real time. The device was tested and verified for accuracy, linearity, hysteresis and repeatability. Preliminary use of the device with athletes in training was performed to verify its potential, but much more work is needed to demonstrate its capabilities to improve training quality. In addition, the device has strong potential in the medical field for the rehabilitation of injuries, whether climbingrelated or not. To this end, one of the prototypes has been installed at the world-leading Klinikum Bamberg in Germany under the direction of renowned orthopedic hand surgeon Dr. Volker Schöffl, wherein such studies will be performed. Useful results on its ability to enhance treatment are forthcoming. Furthermore, ongoing studies will incorporate the device to accurately measure the rate of force development so that enhanced training protocols can be developed to improve this critical characteristic in climbers.

Acknowledgments: Funds for this work were provided by the Department of Engineering Mechanics, US Air Force Academy.

Conflicts of Interest: The authors declare no conflict of interest. The funding sponsors had no role in the design of the study; in the collection, analyses, or interpretation of data; in the writing of the manuscript, and in the decision to publish the results.

\section{References}

1. Baláš, J.; Pecha, O.; Martin, A.J.; Cochrane, D. Hand-arm strength and endurance as predictors of climbing performance. Eur. J. Sport Sci. 2012, 12, 16-25.

2. Anderson, M.; Anderson, M. A novel tool and training methodology for improving finger strength in rock climbers. Procedia Eng. 2015, 112, 491-496.

3. Bourne, R.; Halaki, M.; Vanwanseele, B.; Clarke, J. Measuring Lifting Forces in Rock Climbing: Effect of Hold Size and Fingertip Structure. J. Appl. Biomech. 2011, 27, 40-46.

4. Medernach, J.P.; Kleinöder, H.; Lötzerich, H.H. Fingerboard in Competitive Bouldering: Training Effects on Grip Strength and Endurance. J. Strength Cond. Res. 2015, 29, 2286-2295.

5. Great Trango Holdings Inc. Rock Prodigy Training Center. Available online: http://www.trango.com/p232-rock-prodigy-training-center-by-trango.aspx (accessed on 15 January 2016). 
6. Anderson, M.L.; Anderson, M.; Sanders, A. An innovative hangboard design to improve finger strength in rock climbers. Procedia Eng. 2016, 147, 269-274.

7. Anderson, M.; Anderson, M. The Rock Climber's Training Manual; Fixed Pin: Broomfield, CO, USA, 2014.

8. Romero, V.E. A Physiological Model for Rock Climbing-Today and Beyond. In Proceedings of the 3rd International Rock Climbing Research Congress, Telluride, CO, USA, 5-7 August 2016.

(C) 2018 by the authors; Licensee MDPI, Basel, Switzerland. This article is an open access article distributed under the terms and conditions of the Creative Commons Attribution (CC BY) license (http://creativecommons.org/licenses/by/4.0/). 